THE SHORT CALIPER SPLINT

Robert F. Patterson, M.D., Knoxville, Tenn.

The short caliper splint herein illustrated was developed by me while in charge of the orthopedic service of the U. S. Army base hospital at Fort Sam Houston, Texas. It has

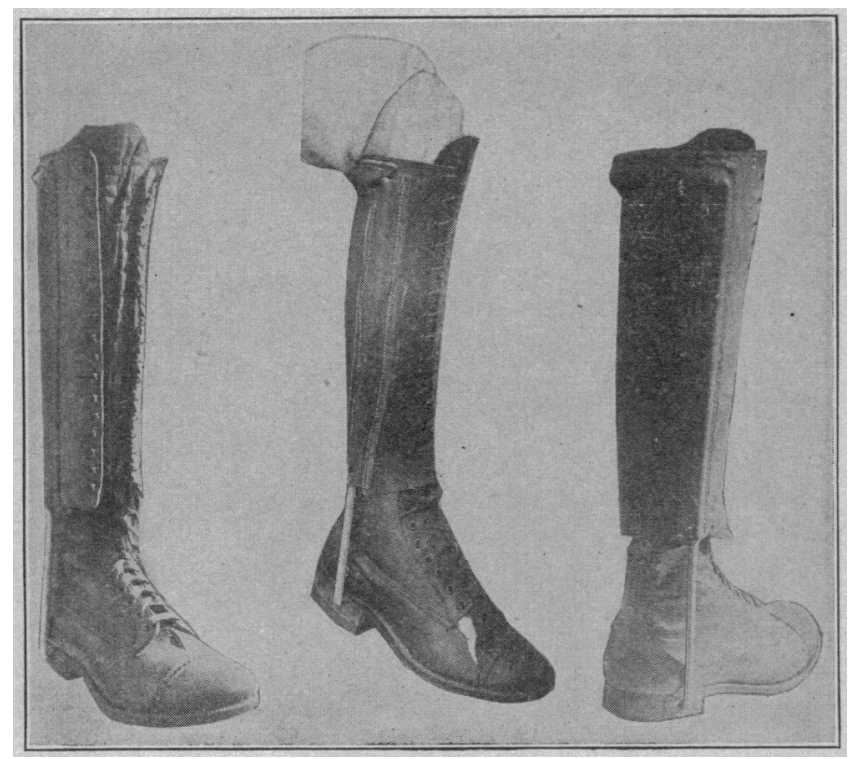

Short caliper splint: front view, in use, and back view.

proved to be uniformly useful in $(a)$ ununited fractures below the knee; (b) weak union, to guard against mishap, and $(c)$ tuberculous or other disease of the ankle or joints of the foot when it is desired to take the weight off of the foot. The splint is intended for the treatment of ambulatory cases.

The right angled portion at the lower end is inserted into the hole in the shoe, as in the application of the long caliper splint. The shoe is now pulled on until the heel lacks about 1 inch of coming in contact with the bottom of the shoe. Next the leather cuff is snugly laced up, and following this the shoe is laced as tightly as can be comfortably borne, after the weight has been borne on the splint. The heel should now just clear the shoe, or rest lightly on it, depending on how much weight it is desired that the foot carry.

If the leg is tender, the cuff can be lined with a pad of saddler's felt glued in place. Ordinarily the patient soon becomes accustomed to the appliance, and prefers no lining except moleskin.

It will be seen that the principle is the same as that of the long caliper, except that this splint takes its bearing surface, as in the case of an artificial leg, from the head of the tibia and fibula, and to a less extent from the entire surface of the leg beneath the cuff, owing to the shape of the leg.

This appliance has these advantages over the long caliper in fractures below the knee: (a) It its inconspicuous, and can be worn under the trousers; (b) it leaves the knee free and thereby prevents stiffness due to immobilization of this joint, and $(c)$ in addition to removing the weight from a fractured bone, it acts as an effectual splint through the medium of the close fitting leather cuff, reinforced by the side irons.

\section{AN IMPROVED DEVICE FOR ARNOLD STERILIZATION *}

\author{
William H. Barnes, A.M., Berkeley, Calif.
}

Large classes in bacteriology necessitate the provision of a good deal of modern and efficient equipment. One of the difficulties with which we have had to contend is the provision of a sufficient number of usable sterilizers, particularly gas heated sterilizers. Although we have enough when they are all in running order, keeping them in good repair has given rise to no little trouble and expense. The gas backfures, and burns in the air chamber of the burner where the air and gas are mixed. This gives less heat units per unit of gas consumed, and results in the production of soot, which collects on the support below and which is scattered about the room. The reservoirs require frequent attention lest they become dry and burn out, notwithstanding the fact that in our laboratory they are filled automatically by siphons, an arrangement devised by Professor Hall. ${ }^{1}$ The siphons cccasionally clog or become empty and fail to maintain a constant water level.

The device here described, which was installed recently in our laboratory, eliminates these difficulties, reduces the cost of maintaining the process of sterilization, and shortens it.

This device, which is shown in the accompanying illustration, consists of a separate one-half inch pipe $(t p)$, with a steam valve $(v)$ carrying live steam from a central source, supplied by the main line steam pipe $(S)$, to the main compartment $(C)$ of each Arnold. A small opening, one-half inch in diameter, is cut in the base $(b)$ of the main compartment $(C)$, and the tapping pipe $(t p)$ is made with an elbow to turn vertically upward into the opening just below the perforated shelf. When the steam is turned on, the temperature immediately rises to 99 or $100 \mathrm{C}$., never going above $100 \mathrm{C}$. This result obtains because the compartment $(C)$ of the Arnold is not air-tight, and thus the steam cannot be confined under pressure. Part of the condensed steam falls into the reservoir $(R)$ below, thus requiring that it be

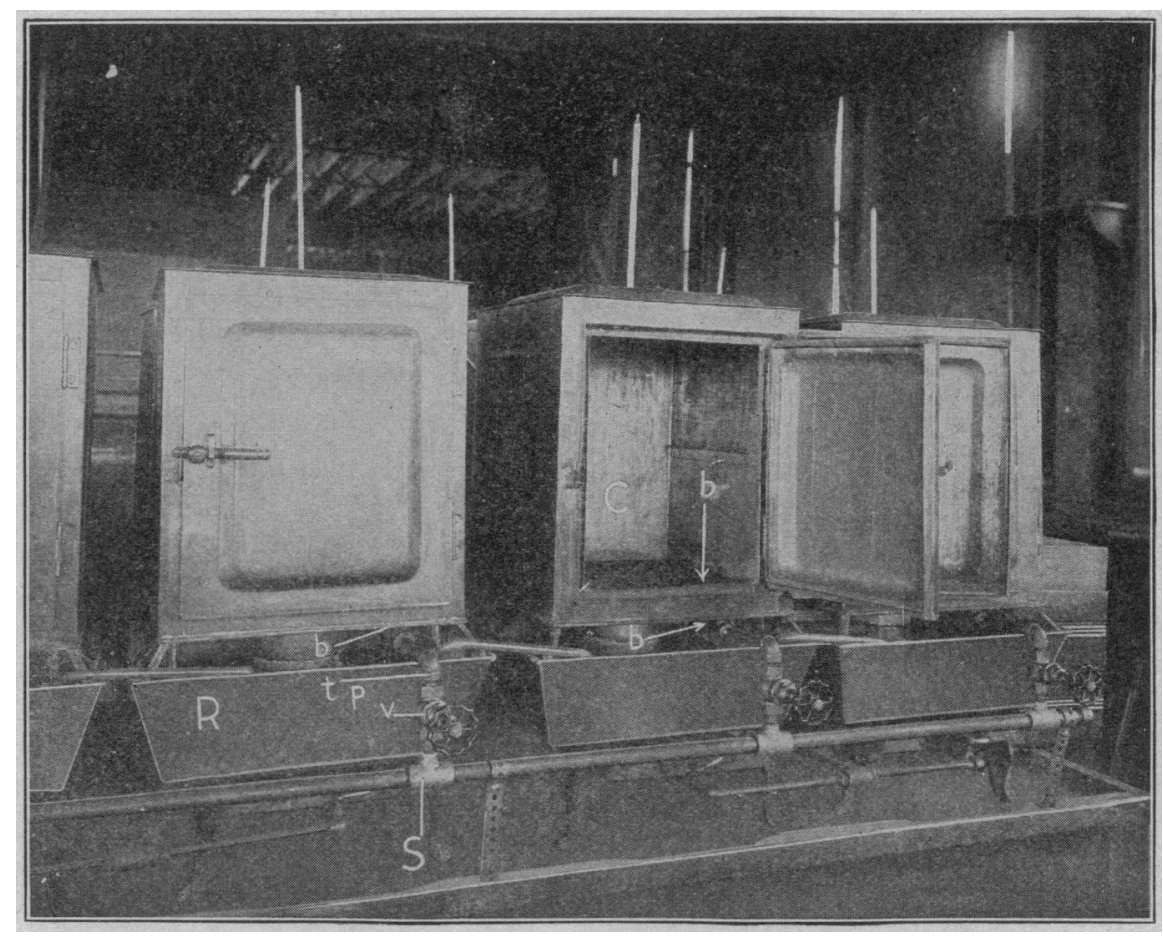

An improved device for Arnold sterilization.

drained instead of being filled with water. Drainage is accomplished very easily by a connection with a nearby drain pipe.

* From the Department of Pathology and Bacteriology. University of California.

1. Hall, T. C.: Automatic Water Level for Arnold Sterilizers, T. Bacteriol. 3:7 (Jan.) 1918. 